\title{
Diversity of Glycoside Hydrolase 10 Family Xylanases Found in Rumen Metagenome and Selection of Sequences with Biotechnological Potential
}

\section{Gabriella Cavazzini Pavarina ${ }^{1}$, Natália Sarmanho Monteiro Lima ${ }^{1,2}$, Claudio Damasceno Pavani ${ }^{1}$, Eliana Gertrudes de Macedo Lemos ${ }^{1,2}$, João Martins Pizauro Junior ${ }^{1}$ and Adriano Marques Gonçalves ${ }^{3,4 *}$}

${ }^{1}$ Technology Department, Sao Paulo State University (Unesp), Faculty of

Agricultural and Veterinary Sciences, Brazil

${ }^{2}$ Molecular Biology Laboratory, Bioenergy Research Institute (IPBEN), Jaboticabal,

Sao Paulo, Unesp, Brazil

${ }^{3}$ Department of Biological and Health Sciences, University of Araraquara

(UNIARA), Brazil

${ }^{4}$ Department of Biochemistry and Organic Chemistry, Chemistry Institute, Sao

Paulo State University (UNESP), Brazil

*Corresponding Author: Adriano Marques Gonçalves, Professor, Department of Biological and Health Sciences, University of Araraquara (UNIARA), Araraquara, SP, Brazil.
Received: June 01, 2021

Published: June 17, 2021

(C) All rights are reserved by Adriano

Marques Gonçalves., et al.

\begin{abstract}
Metagenomics is an important tool for mining and discovering new enzymes, making it possible to explore the diversity of environments. Therefore, this strategy can be used to prospect for xylanases, which degrades lignocellulosic biomass, an important source of renewable energy of great economic interest. The aim of the work was to identify and investigate the diversity of xylanases of the GH10 family present in the rumen metagenome of Nelore cattle and to prospect molecules with good potential for biotechnological application through in silico analyzes. Pfam was used for the initial selection of GH10 sequences, then the physical and chemical parameters were computed using the ProtParam tool, SignalP-5.0 server was used to predict signal peptides and cleavage location, transmembrane helices prediction was made in TMHMM server, version 2.0 and domain annotation was performed with dbCAN meta server. In addition, identity comparison was performed with NCBI BLAST webtool, sequences were aligned with ClustalW and Neighbor-Joining Tree and pairwise analysis were performed. The metagenomics analysis from Nelore cattle rumen returned 38 sequences with the GH10 domain, CE1, GH43 and CBM6 were also identified in these sequences. Analysis of Neighbor-Joining Tree and proteins identity enabled to differentiate 6 groups with, at least, two proteins with identity higher than $70 \%$. Based on the analysis, 13 sequences were considered unappropriated for biotechnological application for either for being unstable or having transmembrane helices. In this sense, based on the in silico analyzes, 25 of the 38 sequences presented good characteristics for in vitro studies. Thus, in addition to the identification of the 38 sequences with GH10 domain, a workflow of in silico methodologies was suggested to assist the selection of sequences that will guide future in vitro studies.
\end{abstract}

Keywords: Metagenomics; Xylanase; In silico Studies; Lignocellulose; Protein Biotechnology; Lignocellulose Degrading Enzymes 


\section{Introduction}

The use of metagenomic databases is valuable for discovering new enzymes making it possible to explore the diversity of environments [1,2]. In this way, this application presents itself as an alternative pathway that helps and enriches the search for new genes related to degradation of lignocellulosic material. Metagenomics provides industry an unprecedented chance to bring diverse biomolecules to industrial application $[3,4]$.

Lignocellulosic biomass, an important source of renewable energy, has gained prominence of great economic interest [5]. In this sense, there are highly efficient microbial communities in the hydrolysis of lignocellulose, such as the digestive system of termites and ruminants $[6,7]$. The degradation of plant biomass by ruminants is a consequence of the symbiosis between these and the numerous microorganisms, present in the ruminal cavity, that specialize for this purpose [7]. The metagenomics of the rumen environment allows both understanding of the functioning of this microenvironment, as well as the identification of new enzymes for the hydrolysis of lignocellulosic biomass [8-10].

Among hemicellulose degrading enzymes, xylanases are responsible for cleaving xylans, the most abundant hemicellulose in nature. The biodegradation of xylans involves the action of several hydrolytic enzymes, those that act on the branches (acetyl xylan esterease, $\alpha$-D-glucoronidase, $\alpha$-L-arabinofuranosidase), and those acting in the main chain (endo-1,4- $\beta$-xylanases EC 3.2.1.8 and $\beta$-xilosidase, EC 3.2.1.37) [11], leading to the release of xylooligosaccharides, which will be hydrolyzed by the other hemicellulases. Hemicellulases, together with auxiliary enzymes, increase the yield of monosaccharide generation in the enzymatic hydrolysis process of the lignocellulosic complex [12]. Within the group of hemicellulases, xylanases are used in industry in xylooligosaccharides production processes to obtain xylitol [15], production of fermentable sugars for the production of second generation ethanol [13] and paper production [11].

The heterologous expression of proteins using Escherichia coli is one of the most common systems when high productivity of recombinant proteins is desired, E. coli is called a "microbial factory", due to its easy handling, low production cost and high efficiency [16]. Even though it is highly advantageous, it depends on the characteristics of each gene, often making the process difficult [17], and some strategies can be taken to increase the efficiency of the process and avoid further problems. For example, some proteins, such as membrane proteins and proteins of larger molecular size, are often not expressed in E. coli, or when they are, they form inclusion bodies [18]. The presence of signal peptides can also cause problems in the production of heterologous proteins, often leading to insoluble molecules [19]. Therefore, the use of tools that provide prior knowledge of the gene is extremely important for the overexpression of recombinant proteins. The use of bioinformatics through in silico prediction significantly increases the chances of protein expression success [20]. Most of these tools offer an alternative of optimization, restriction sites, removal of harmful codons or nucleotides, in addition, the great advantage is that they are often available in online servers [21].

In this sense, the present work, using in silico analysis, investigated the diversity of GH10 xylanases in the rumen of Nellore cattle and the characteristics of the identified sequences. These studies were carried out aiming to differentiate and indicate the sequences with the greatest potential for biotechnological application. Additionally, a workflow was proposed to systematize criteria that are essential for the selection of sequences with greater biotechnological potential.

\section{Materials and Methods \\ Identification of xylanases}

The metagenomic data used for prospecting the sequences were obtained from the sequencing llumina HiScanSQ sequencing data set from Nelore cattle rumen (public data access: SRX818104), which assembly and annotation of genes were stored in the metagenomic database of the Laboratory of Biochemistry and Plant Microorganisms (LBMP). For this purpose, a reference sequence of endo- $\beta-1,4$-xylanase from the GH10 family, obtained in The American database of the National Center for Biotechnology Information (NCBI) was used to infer the xylanases sequences, then the sequences were submitted to the database eggNOG orthologist [22]. The ORFs (open reading frames) were selected with an e-value of -30, aligned with ClustalW [23], and validated for enzymatic domains in the Pfam database [24].

Prediction of physical and chemical parameters, signal peptides, transmembrane helices and domains

The physical and chemical parameters were computed using the ProtParam tool [25], SignalP-5.0 server was used to predict signal peptides and cleavage location [26], transmembrane helices prediction was made in TMHMM server, version 2.0 [27] and domain annotation was performed with dbCAN meta server [28]. 
Sequence alignment and estimated divergence between sequences

The sequences were organized in a FASTA file and, in order to estimate identities between sequences, a protein-protein BLAST was used to align the obtained sequences [29]. In addition, sequences were aligned with NCBI patent database using the same BLAST tool.

Also, the sequences were aligned with multiple alignment tool ClustalW with Clustal X (version 2.0) software [30]. After the ClustalW alignment the number of amino acid differences per sequence from between sequences were calculated. Standard error estimates were obtained by a bootstrap procedure (1000 replicates). This analysis involved 38 amino acid sequences containing gh10 domain. All ambiguous positions were removed for each sequence pair (pairwise deletion option). There were a total of 1410 positions in the final dataset. Evolutionary analyses were conducted in MEGA X [31].

Next, a Neighbor-Joining tree was inferred to evaluate the relationship between the protein sequences [32]. The bootstrap consensus tree inferred from 1000 replicates was taken to repre- sent the relation of the sequences analyzed [33]. Branches corresponding to partitions reproduced in less than 75 bootstrap replicates were collapsed. Sequences distances were computed using the number of differences method [34] and are in the units of the number of amino acid differences per sequence. This analysis involved the 38 amino acid sequences. All ambiguous positions were removed for each sequence pair (pairwise deletion option). There were a total of 1410 positions in the final dataset. The referred analysis was conducted in MEGA X [31]. The final tree was analyzed and generated with ITol (Interactive Tree of Life) tool [35].

\section{Results and Discussion}

The metagenomics analysis from Nelore cattle rumen returned 38 sequences with the GH10 domain, additionally, the sequences may contain other domains (Table 1), this type of analysis is important to infer GH10 diversity in this complex environment. Besides, other domain recognition may be used to choose between sequences to be investigated in further steps, to clone and express the molecules with best biotechnology potential. Xylanases have broad use in industrial processes [11,13-15], in this sense, this kind of study is important to identify new molecules, in order to improve enzyme utilization in industrial and biotechnological procedures.

\begin{tabular}{|c|c|c|c|c|c|c|c|c|c|c|}
\hline ID & $\begin{array}{c}\text { Sequence } \\
\text { Length } \\
\text { (aa) }\end{array}$ & $\begin{array}{l}\text { Molecular } \\
\text { Weight } \\
\text { (kDa) }\end{array}$ & Domains & $\begin{array}{l}\text { Instability } \\
\text { Index* }\end{array}$ & TМНММ & pI & $\begin{array}{c}\% \text { of } \\
\text { positive } \\
\text { aa** }\end{array}$ & $\begin{array}{c}\begin{array}{c}\% \text { of } \\
\text { negative }\end{array} \\
\text { aa*** }\end{array}$ & $\begin{array}{l}\text { Aliphatic } \\
\text { Index }\end{array}$ & SignalP**** \\
\hline Xyl_01 & 368 & 41.62 & GH10(24-365) & $32.36 / \mathrm{Y}$ & 0 & 7,06 & 12.2 & 12.2 & 88.80 & $\mathrm{Y}(1-21)$ \\
\hline Xyl_02 & 719 & 81.28 & $\begin{array}{c}\text { GH10(34- } \\
364)+\mathrm{CE} 1(503-715)\end{array}$ & $32.41 / Y$ & 0 & 6,03 & 11.4 & 12.8 & 67.97 & $\mathrm{Y}(1-20)$ \\
\hline Xyl_03 & 387 & 44.52 & GH10(33-383) & $28.02 / \mathrm{Y}$ & 0 & 5,22 & 11.4 & 15.8 & 83.10 & $\mathrm{~N}$ \\
\hline Xyl_04 & 724 & 82.10 & $\begin{array}{c}\text { GH10(34- } \\
\text { 365)+CE1(505-720) }\end{array}$ & $34.25 / Y$ & 0 & 6,44 & 12.2 & 12.8 & 72.32 & $\mathrm{Y}(1-20)$ \\
\hline Xyl_05 & 753 & 85.29 & $\begin{array}{c}\text { GH10(26- } \\
346)+\mathrm{CE} 1(537-748)\end{array}$ & $41.85 / \mathrm{N}$ & 0 & 8,53 & 12.6 & 11.8 & 62.59 & $Y(1-24)$ \\
\hline Xyl_06 & 731 & 82.81 & $\begin{array}{c}\text { GH10(33- } \\
\text { 367)+CE1(507-728) }\end{array}$ & $34.69 / Y$ & 0 & 7,89 & 12.4 & 12.2 & 71.78 & $Y(1-20)$ \\
\hline Xyl_07 & 711 & 80.42 & $\begin{array}{c}\text { GH10(36- } \\
369)+ \text { CE1(509-687) }\end{array}$ & $32.92 / Y$ & 1 & 6,44 & 12.1 & 12.8 & 69.93 & $\mathrm{Y}(1-23)$ \\
\hline Xyl_08 & 723 & 81.65 & $\begin{array}{c}\text { GH10(35- } \\
368)+ \text { CE1(507-719) }\end{array}$ & $30.34 / Y$ & 0 & 6,21 & 10.9 & 12.0 & 65.84 & $\mathrm{Y}(1-21)$ \\
\hline Xyl_09 & 727 & 82.39 & $\begin{array}{c}\text { GH10(34- } \\
368)+ \text { CE1(508-723) }\end{array}$ & $35.81 / Y$ & 0 & 6,38 & 12.4 & 13.2 & 74.04 & $\mathrm{Y}(1-21)$ \\
\hline Xyl_10 & 368 & 41.91 & GH10(25-365) & $26.95 / Y$ & 0 & 6,72 & 12.2 & 12.5 & 90.35 & $Y(1-22)$ \\
\hline Xyl_11 & 754 & 84.48 & $\begin{array}{c}\text { GH10(35- } \\
360)+ \text { CE1(499-750) }\end{array}$ & $38.74 / Y$ & 0 & 5,69 & 10.5 & 12.7 & 70.04 & $\mathrm{Y}(1-22)$ \\
\hline
\end{tabular}


Diversity of Glycoside Hydrolase 10 Family Xylanases Found in Rumen Metagenome and Selection of Sequences with Biotechnological Potential

\begin{tabular}{|c|c|c|c|c|c|c|c|c|c|c|}
\hline Xyl_12 & 368 & 42.19 & GH10(27-365) & $30.16 / Y$ & 0 & 6,21 & 11.7 & 10.6 & 80.65 & $\mathrm{Y}(1-23)$ \\
\hline Xyl_13 & 345 & 39.81 & GH10(22-343) & $36.00 / Y$ & 0 & 6,56 & 12.2 & 12.8 & 77.74 & $Y(1-20)$ \\
\hline Xyl_14 & 652 & 72.26 & GH10(60-386) & $29.05 / Y$ & 1 & 6,36 & 12.0 & 12.4 & 77.81 & $Y(1-25)$ \\
\hline Xyl_15 & 744 & 84.43 & $\begin{array}{c}\text { GH10(23- } \\
347)+ \text { CE1(528-738) }\end{array}$ & $43.47 / \mathrm{N}$ & 0 & 5,86 & 10.8 & 12.4 & 68.84 & $Y(1-21)$ \\
\hline Xyl_16 & 379 & 42.99 & GH10(35-376) & $39.09 / Y$ & 1 & 7,15 & 12.1 & 12.1 & 83.88 & $\mathrm{Y}(1-33)$ \\
\hline Xyl_17 & 368 & 41.55 & GH10(24-365) & $36.65 / Y$ & 0 & 7,06 & 11.1 & 11.1 & 86.71 & $\mathrm{Y}(1-21)$ \\
\hline Xyl_18 & 728 & 82.92 & $\begin{array}{c}\text { GH10(34- } \\
370)+ \text { CE1(509-724) }\end{array}$ & $37.57 / Y$ & 1 & 6,76 & 12.9 & 13.2 & 72.72 & $Y(1-22)$ \\
\hline Xyl_19 & 714 & 81.61 & $\begin{array}{c}\text { GH10(24- } \\
355)+ \text { CE1(498-710) }\end{array}$ & $37.72 / \mathrm{Y}$ & 0 & 6,21 & 12.6 & 13.7 & 70.57 & $Y(1-20)$ \\
\hline Xyl_20 & 727 & 82.22 & $\begin{array}{c}\text { GH10(34- } \\
368)+ \text { CE1(508-723) } \\
\end{array}$ & $35.98 / Y$ & 0 & 6,29 & 12.5 & 13.3 & 72.43 & $\mathrm{Y}(1-22)$ \\
\hline Xyl_21 & 746 & 84.00 & $\begin{array}{c}\text { GH10(37- } \\
371)+ \text { CE1 }(509-728)\end{array}$ & $40.04 / \mathrm{N}$ & 0 & 6,89 & 11.7 & 11.9 & 72.96 & $Y(1-23)$ \\
\hline Xyl_22 & 645 & 73.46 & $\begin{array}{c}\text { GH10(33- } \\
366)+\mathrm{CE} 1(506-639)\end{array}$ & $35.18 / Y$ & 0 & 7,98 & 12.6 & 12.2 & 70.59 & $\mathrm{Y}(1-20)$ \\
\hline Xyl_23 & 644 & 75.85 & $\begin{array}{c}\text { GH10(8- } \\
343)+ \text { CE1 }(483-634)\end{array}$ & $37.20 / Y$ & 0 & 6,33 & 13.2 & 14.1 & 68.99 & $\mathrm{~N}$ \\
\hline Xyl_24 & 433 & 49.78 & GH10(35-369) & $38.62 / Y$ & 1 & 8,35 & 14.5 & 13.9 & 74.32 & $\mathrm{Y}(1-24)$ \\
\hline Xyl_25 & 382 & 43.20 & GH10(37-373) & $28.44 / Y$ & 0 & 9,37 & 15.4 & 11.8 & 77.15 & $Y(1-26)$ \\
\hline Xyl_26 & 501 & 57.39 & GH10(21-355) & $39.42 / Y$ & 0 & 6 & 12.0 & 13.8 & 65.35 & $\mathrm{~N}$ \\
\hline Xyl_27 & 725 & 82.40 & $\begin{array}{c}\text { GH10(34- } \\
\text { 370)+CE1(512-722) }\end{array}$ & $38.70 / Y$ & 0 & 6,26 & 12.4 & 13.2 & 68.61 & $\mathrm{Y}(1-21)$ \\
\hline Xyl_28 & 723 & 82.04 & $\begin{array}{c}\text { GH10(30- } \\
360)+ \text { CE1 }(507-719)\end{array}$ & $38.91 / Y$ & 0 & 7,02 & 12.2 & 12.3 & 74.44 & $Y(1-19)$ \\
\hline Xyl_29 & 385 & 44.51 & GH10(49-383) & $37.63 / Y$ & 1 & 8,03 & 13.0 & 12.5 & 67.12 & $\mathrm{Y}(1-35)$ \\
\hline Xyl_30 & 726 & 82.52 & $\begin{array}{c}\text { GH10(33- } \\
367)+ \text { CE1 }(507-722) \\
\end{array}$ & $32.32 / Y$ & 0 & 7,24 & 12.9 & 12.9 & 71.43 & $Y(1-19)$ \\
\hline Xyl_31 & 748 & 85.03 & $\begin{array}{c}\text { GH10 }(42- \\
379)+ \text { CE1 }(518-743)\end{array}$ & $33.82 / Y$ & 1 & 6,42 & 12.4 & 13.1 & 70.40 & $\mathrm{Y}(1-29)$ \\
\hline Xyl_32 & 418 & 47.85 & GH10(3-327) & $35.54 / Y$ & 0 & 5,67 & 11.5 & 14.1 & 70.89 & $\mathrm{~N}$ \\
\hline Xyl_33 & 389 & 45.23 & GH10(36-371) & $36.33 / Y$ & 1 & 8,47 & 14.1 & 13.1 & 65.42 & $\mathrm{Y}(1-22)$ \\
\hline Xyl_34 & 726 & 82.27 & $\begin{array}{c}\text { GH10(33- } \\
367)+ \text { CE1(507-722) }\end{array}$ & $36.21 / \mathrm{Y}$ & 0 & 7,9 & 13.1 & 12.8 & 70.25 & $Y(1-20)$ \\
\hline Xyl_35 & 432 & 49.32 & GH10(44-427) & $44.81 / \mathrm{N}$ & 0 & 5,51 & 10.9 & 13.2 & 71.83 & $\mathrm{~N}$ \\
\hline Xyl_36 & 691 & 77.77 & $\begin{array}{c}\text { GH10(17- } \\
302)+ \text { CBM6(446-551) }\end{array}$ & $29.65 / Y$ & 0 & 5,33 & 9.7 & 12.2 & 68.08 & $\mathrm{~N}$ \\
\hline Xyl_37 & 849 & 95.33 & $\begin{array}{c}\text { GH43_7(26- } \\
295)+ \text { GH10(500-849) }\end{array}$ & $42.50 / \mathrm{N}$ & 0 & 4,99 & 9.7 & 12.8 & 75.18 & $\mathrm{Y}(1-22)$ \\
\hline Xyl_38 & 305 & 35.03 & GH10(1-302) & $32.88 / Y$ & 0 & 6,03 & 12.1 & 15.8 & 79.02 & $\mathrm{~N}$ \\
\hline
\end{tabular}

Table 1: Predicted physico-chemical parameters, domains, transmembrane helices and signal peptide of the 38 identified GH10 sequences.

${ }^{*}$ Y refers to stable proteins and $\mathrm{N}$ refers to unstable proteins. ${ }^{* *}$ Percentage of positively charged residues (Arg and Lys). ${ }^{* * *}$ Percentage of negatively charged residues (Asp and Glu). ${ }^{* * * *}$ Y indicates presence of signal peptide and $\mathrm{N}$ indicates absence of signal peptide. 
In order to characterize and analyze the sequences returned by prospecting, in silico analyzes were carried out to search for the most promising molecules to be tested in future in vitro heterologous expression assays. Despite not substituting laboratory tests, in silico analysis contributes to the choice of molecules with the best potential, leading to savings in human and financial resources and time. Thus, a workflow (Figure 1) was used to choose, among the 38 sequences, those with better conditions for heterologous expression.

Figure 1: Workflow for protein sequence selection.

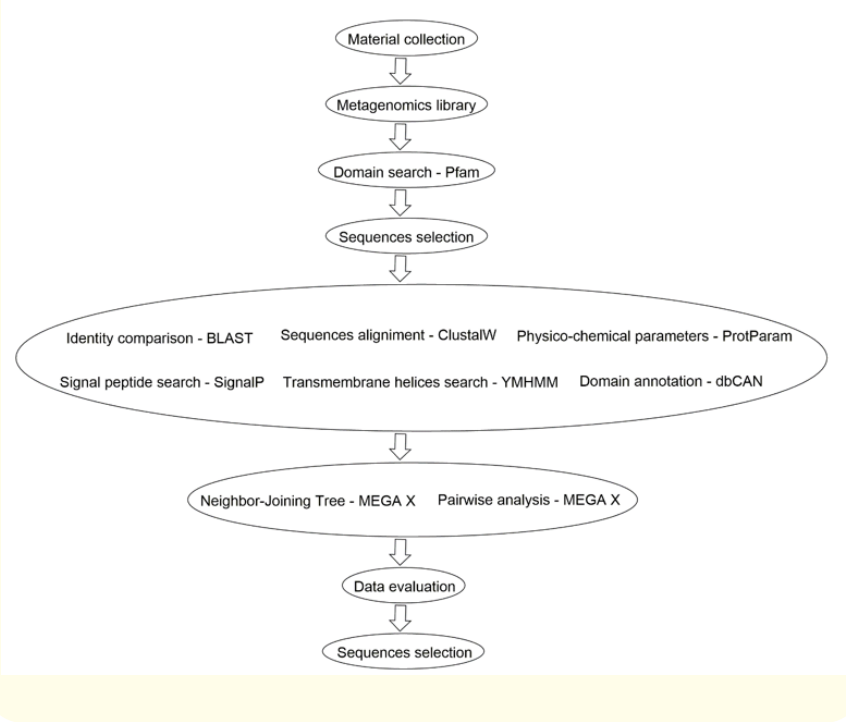

The alignment of the 38 sequences, followed by an analysis by Neighbor-Joining Tree, made it possible to establish relationships between the sequences. When Neighbor-Joining Tree (Figure 2) and proteins identity (Table 2) are compared it is possible to differentiate 6 groups with, at least, two proteins with identity higher than 70\%. These groups are divided in branches with Xyl_23 to Xyl_22 and Xyl_33 to Xyl_07 (17 proteins with high identity); branch with Xyl_38 to Xyl_10 (5 proteins with high identity); Xyl_37 and Xyl_03; Xyl_11 and Xyl_32; Xyl_02 and Xyl_08; Xyl_28 and 19. Proteins Xyl_35, Xyl_13, Xyl_05, Xyl_15, Xyl_12, Xyl_36, Xyl_14 and Xyl_25 do not have high identity with any of the identified sequences (Table 2).
These groups, together with the degree of identity between the sequences, provide important relationships for knowing the diversity of proteins that may have different properties. Another analysis that contributed in this regard was the BLAST of the 38 sequences against the NCBI patent database. The results in table 4 demonstrated the presence of sequences with up to $68.79 \%$ identity (Xyl_07 and AKY00359.1). The observed values of identity are not high and do not exclude future studies with the herein identified sequences. In this sense, sequences obtained by metagenomic analysis of rumen can contribute in an important way to the discovery of new molecules with biotechnological potential.

Figure 2: Neighbor-Joining Tree inferred after ClustalW alignment of the 38 identified proteins.

The percentage of replicate trees in which the associated proteins clustered together in the bootstrap test (1000 replicates) are shown next to the branches.

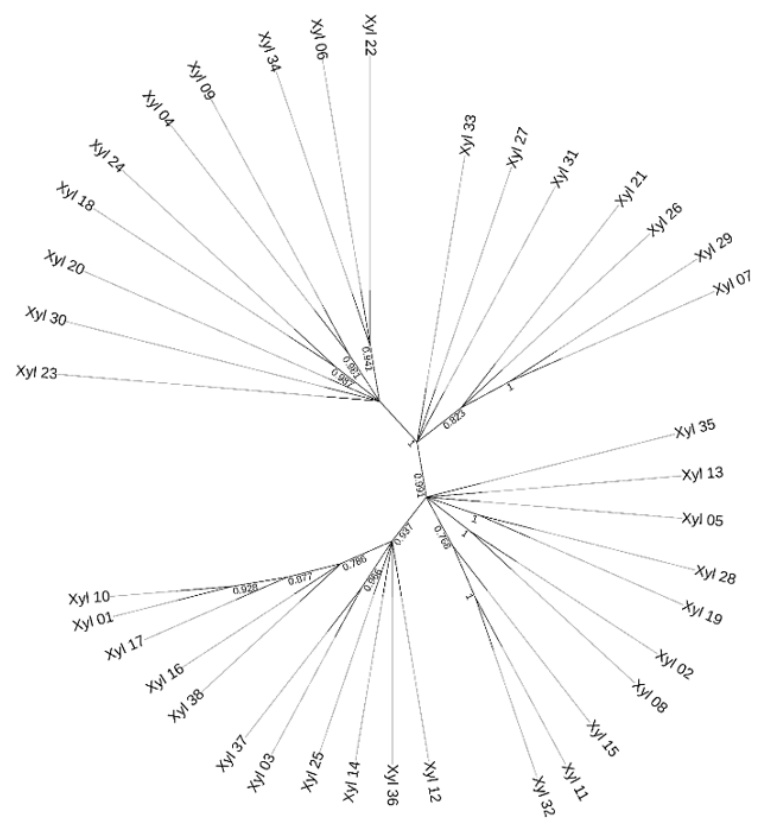




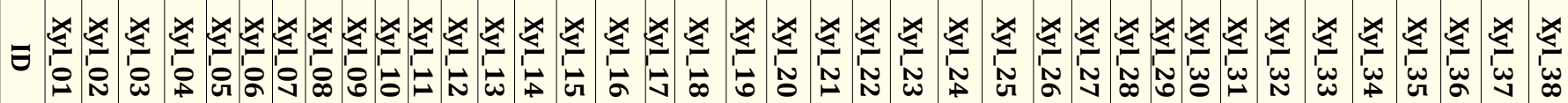

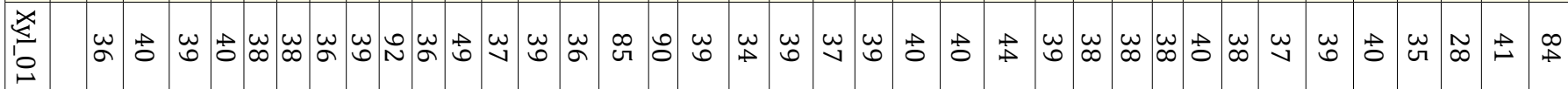

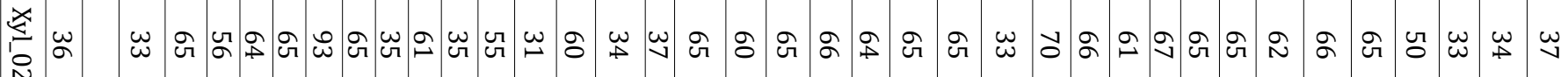

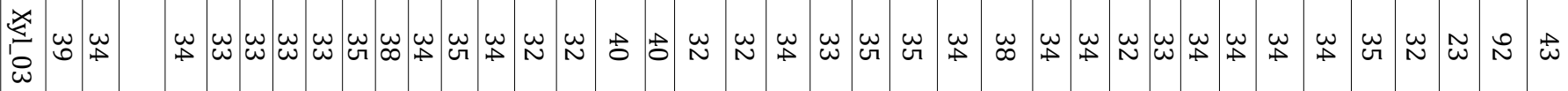

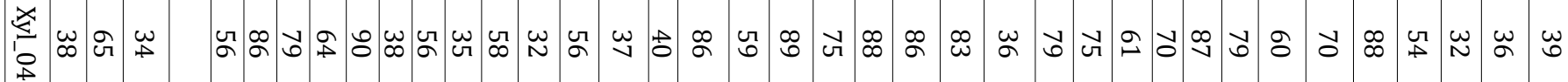

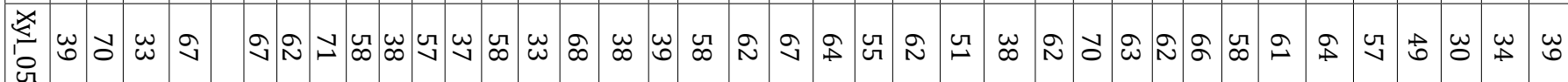

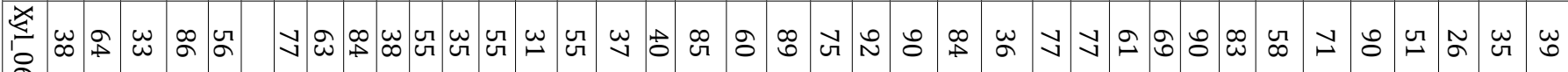

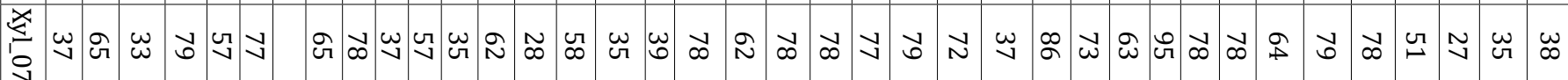

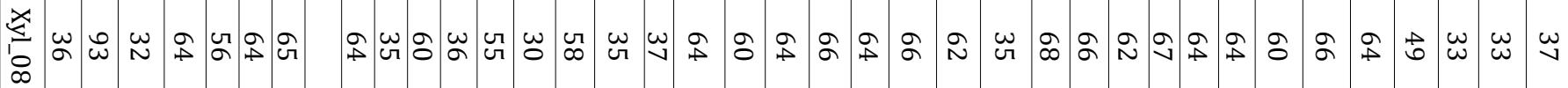

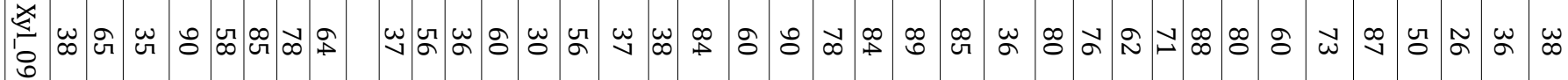

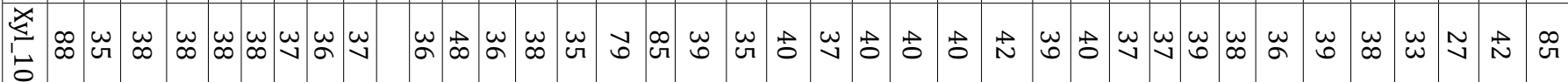

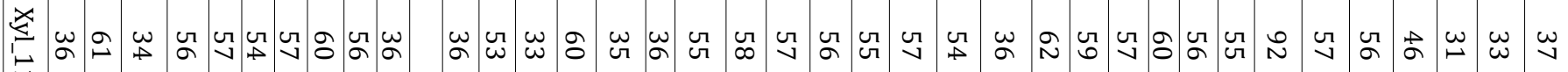

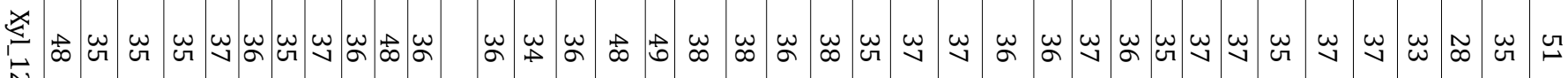

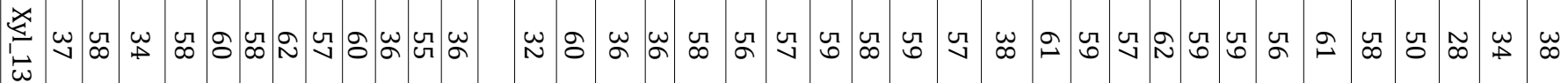

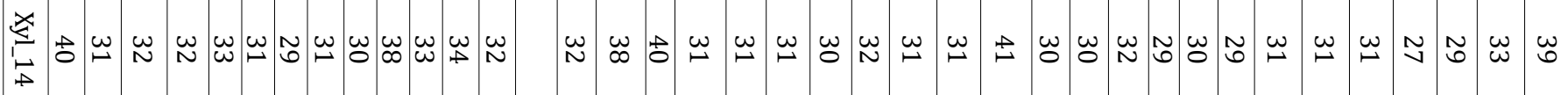

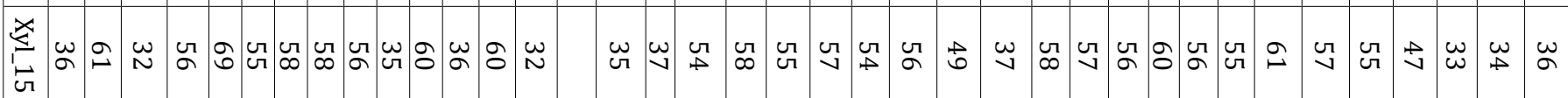

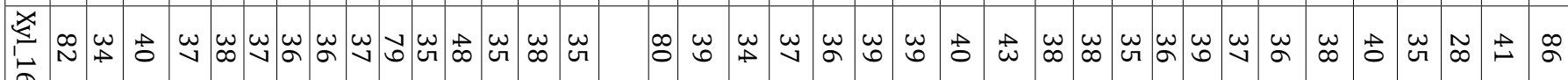

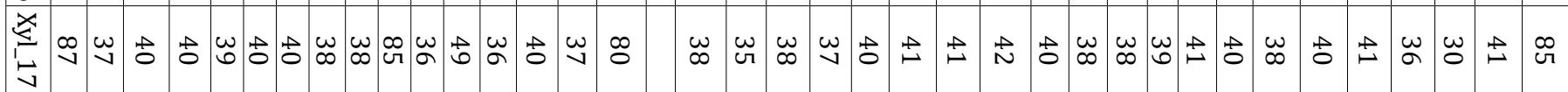




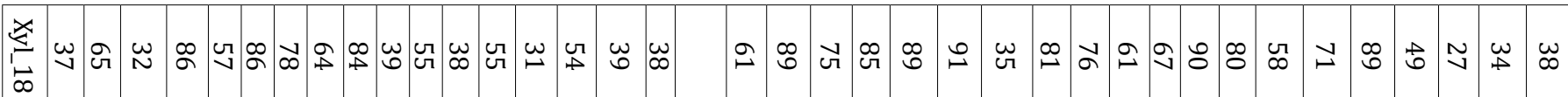

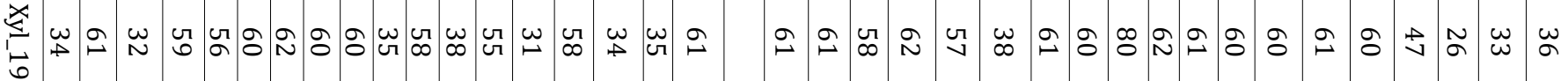

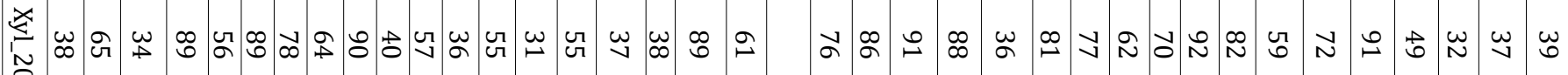

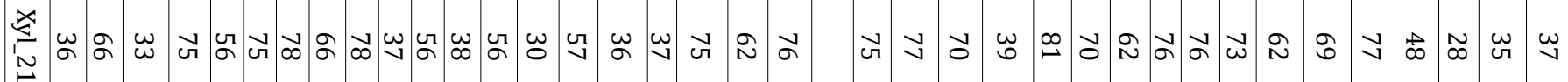

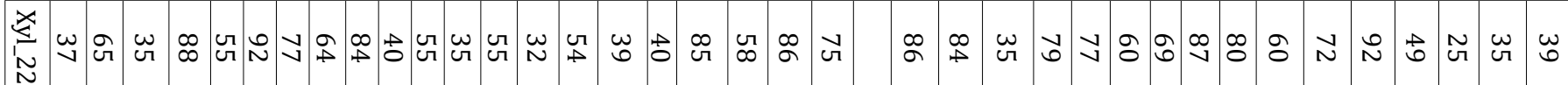

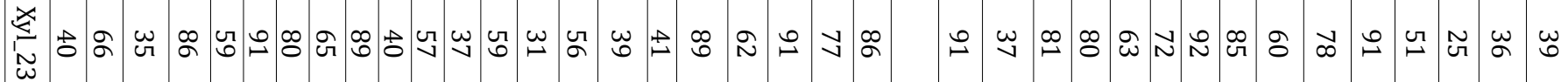

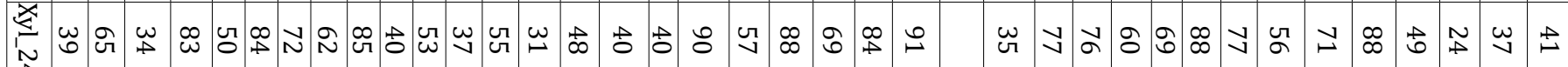

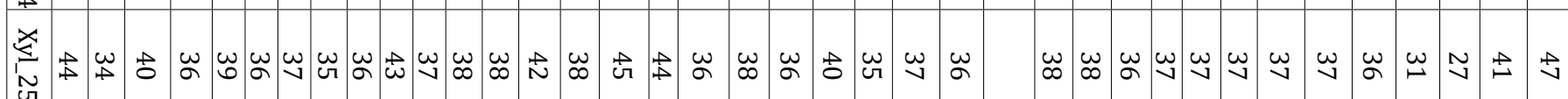

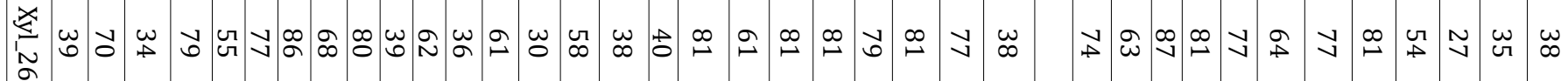

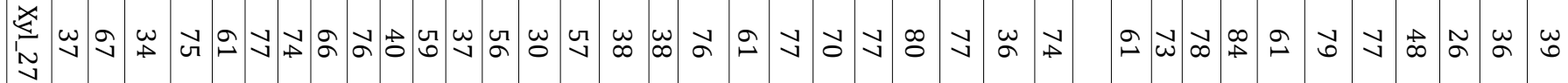

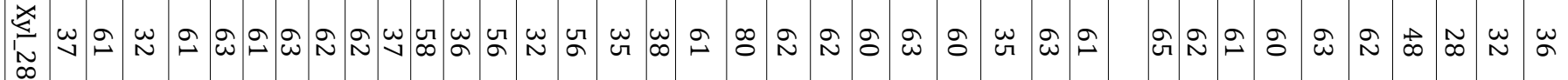

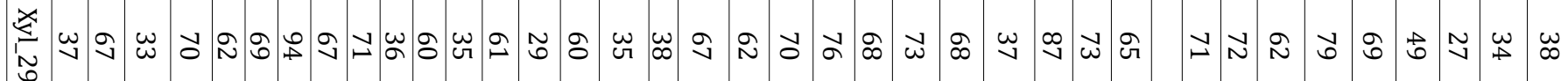

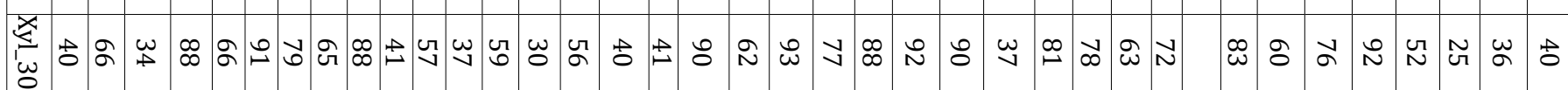

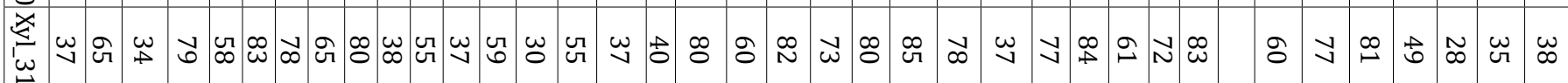

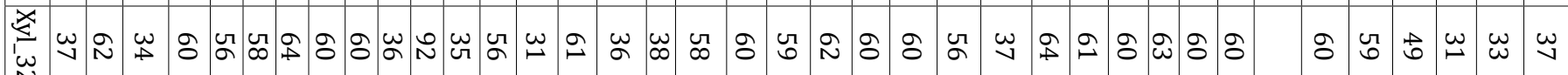

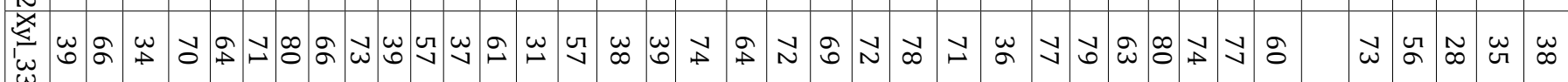

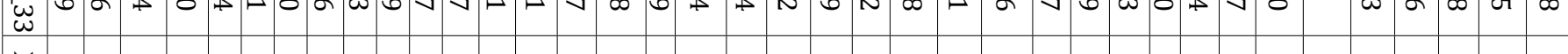

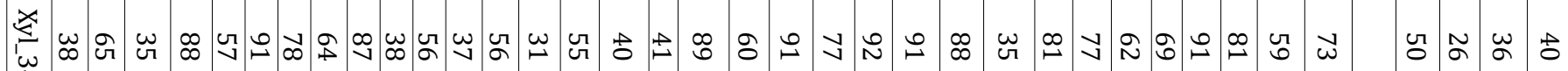

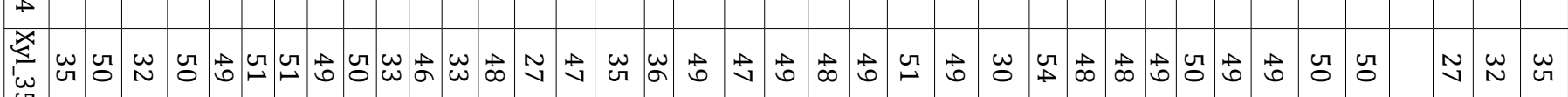




\begin{tabular}{|c|c|c|c|c|c|c|c|c|c|c|c|c|c|c|c|c|c|c|c|c|c|c|c|c|c|c|c|c|c|c|c|c|c|}
\hline w & $\infty$ & & $\tilde{\omega}$ & $\underset{N}{\omega}$ & & 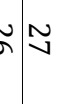 & $\underset{\omega}{\omega}$ & $\tilde{\sigma}$ & $\stackrel{\sim}{\sim})$ & $\begin{array}{c}\sim \\
\sim\end{array}$ & $\infty$ & $\tilde{O}$ & $\underset{\omega}{\omega}$ & $\begin{array}{lll}\infty & \omega \\
\infty\end{array}$ & $\mathcal{V}$ & $\alpha$ & $\stackrel{\omega}{N}$ & $\infty$ & $\tilde{u}$ & $\tilde{v}$ & $\stackrel{\sim}{\perp}$ & $\infty$ & $\mathcal{v}$ & $\hat{\sigma} \hat{\alpha}$ & $\begin{array}{ccc}\sim & \sim \\
\infty\end{array}$ & $\begin{array}{lll}\mathcal{N} & N \\
\mathcal{U}\end{array}$ & $\stackrel{\omega}{\bullet}$ & $\tilde{O}$ & $\tilde{\sigma}$ & $\mathcal{N}$ & & & $\stackrel{\omega}{\sim}$ \\
\hline w & $\boxplus$ & & $\stackrel{\sim}{\sim}$ & $\omega$ & & 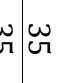 & $\underset{\perp}{\omega}$ & & $\stackrel{\vec{N}}{\omega} \omega_{\omega}$ & $\begin{array}{c}u \\
u \\
u\end{array}$ & $\stackrel{\omega}{\oplus}$ & $\underset{\omega}{\omega}$ & $\underset{\phi}{\omega}$ & $\vec{t} \vec{H}$ & $\stackrel{\omega}{t}$ & $\underset{\omega}{\omega}$ & $\tilde{w}^{\prime}$ & $\begin{array}{l}\omega \\
\omega \\
\sim\end{array}$ & $\mid \begin{array}{l}w \\
\sim \\
\sim\end{array}$ & $\tilde{\sigma}$ & $\underset{v}{v}$ & $\overrightarrow{0}$ & $\underset{\perp}{\omega}$ & \begin{tabular}{c|c}
$\omega$ & $u$ \\
$n$
\end{tabular} & 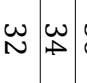 & \begin{tabular}{|c|c}
$\omega$ & $w$ \\
$\sigma$ & $u$
\end{tabular} & $\underset{\omega}{\omega}$ & $\underset{v}{w}$ & $\tilde{\sigma}$ & $\underset{N}{\omega}$ & $\tilde{\omega}$ & & $\vec{\omega}$ \\
\hline ָ. & $\infty$ & & $\vec{\omega}$ & $\tilde{\sigma}$ & & & $\underset{\infty}{\omega}$ & $\begin{array}{l}\omega \\
\infty\end{array}$ & 必 w & ט & $\begin{array}{l}\omega \\
\infty\end{array}$ & $\ddot{\sigma}$ & $\tilde{\sigma}$ & \begin{tabular}{lll}
$\infty$ & $\infty$ \\
\hdashline
\end{tabular} & $\mathfrak{l}_{\infty}^{\infty}$ & $\tilde{\sigma}$ & ڤ્ & $\underset{v}{\omega}$ & $\tilde{\omega}$ & W & $\vec{t}$ & $\stackrel{ \pm}{v}$ & $\begin{array}{l}\omega_{\infty} \\
\end{array}$ & \begin{tabular}{c|c}
$\omega$ & $\mathscr{c}$
\end{tabular} & $\begin{array}{c}\omega \\
\sigma \\
\infty\end{array}$ & $\overrightarrow{0} \mid \begin{array}{c}w \\
\infty\end{array}$ & $\stackrel{w}{v}$ & $\begin{array}{l}\omega \\
\infty\end{array}$ & 8 & 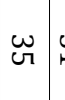 & $\stackrel{\omega}{\omega}$ & & \\
\hline $\begin{array}{l}v \\
\text { va } \\
\text { de }\end{array}$ & 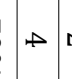 & & $\bullet$ & $\vec{v}$ & 00 & $\vec{s}$ & N & & $\oplus$ & & 0 & 0 & 0 & $A \rightarrow$ & $\vec{r}$ & 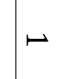 & $\vec{v}$ & $\vec{t}$ & $\vec{r}$ & $\vec{\sigma}$ & $\vec{b}$ & 0 & $\vec{\sigma}$ & $\vec{v}$ & $\bullet \mapsto$ & హ゙ & $\mapsto$ & $\vec{v}$ & $\vec{a}$ & 0 & 0 & & 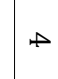 \\
\hline
\end{tabular}

Table 2: Percentage of identity between the 38 sequences identified in Metagenomic analysis and counting of sequences with identity higher than $70 \%$.

In addition to GH10, the analysis of the domains present in the 38 sequences on the dbCAN platform made it possible to identify three other domains (Table 1). The CE1 domain, Carbohydrate esterase family 1 , corresponds to an extensive class of enzymes capable of degrading xylan [36], and is present in most of the identified sequences: Xyl_02; Xyl_04; Xyl_05; Xyl_06; Xyl_07; Xyl_08; Xyl_09; Xyl_11; Xyl_15; Xyl_18; Xyl_19; Xyl_20; Xyl_21; Xyl_22; Xyl_23; Xyl_27; Xyl_28; Xyl_30; Xyl_31 e Xyl_34. The CBM6 domain, carbohydrate-binding modules, is common in xylanases, having an auxiliary role in the hydrolysis of insoluble substrates [37] and was identified in Xyl_36. Xyl_37 contains the GH43 domain, glycoside hydrolase, related on the degradation of hemicellulose and pectin [38]. In this sense, the presence of complementary domains can contribute to the choice of multifunctional molecules, something desired in biotechnological applications [39].

Additionally, ProtParam (Table 1) and Pairwise analysis (Table 3) show that the identified sequences are distinct based on amino acids residues number. According to table 3 the differences between amino acids residues number did not interfere in sequences identity (Table 2) and Neighbor-Joining Tree clustering (Figure 2). Besides, ProtParam returned the prediction of physical-chemical parameters of the identified sequences (Table 1). It was possible to observe the presence of 33 stable proteins (values lower than 40), and the unstable ones were considered inadequate for future studies. The aliphatic index is related to the thermostability of globular proteins, higher values indicate possibly more thermostable pro- teins [25]. This is an important characteristic when concerning biotechnological applications, therefore, sequences Xyl_01, Xyl_03, Xyl_10, Xyl_12, Xyl_16 and Xyl_17 presented values above 80, in the other hand, the lowest observed value was 62.59 for Xyl_05 (Table 1).

In addition, the analysis conducted on the webtool SignalP identified the presence of signal peptide in 31 of the 38 sequences (Table 1). The presence of signal peptide may hinder studies of cloning and expression of proteins in vectors, despite this, signal peptides can be removed when starting in vitro studies, so this is not a determining factor in discarding a particular sequence. Another point considered was the absence of transmembrane helices, since these structures make it difficult to express and purify proteins, in this sense, only 8 of the 38 sequences have this undesired characteristic (Table 1).

According to the excluding criteria (instability index and transmembrane helices) the sequences Xyl_05, Xyl_07, Xyl_14, Xyl_15, Xyl_16, Xyl_18, Xyl_21, Xyl_24, Xyl_29, Xyl_31, Xyl_33, Xyl_35 and Xyl_37 were considered not viable for cloning and expression. Instability index is an excluding criterion since stability is directly related to the production of heterologous enzymes, in systems like E. coli, and unstable proteins can not be applicated in biotechnological sectors. The presence of transmembrane helices in the prediction of the secondary structure may also be a determining factor that makes heterologous production unfeasible, since these 


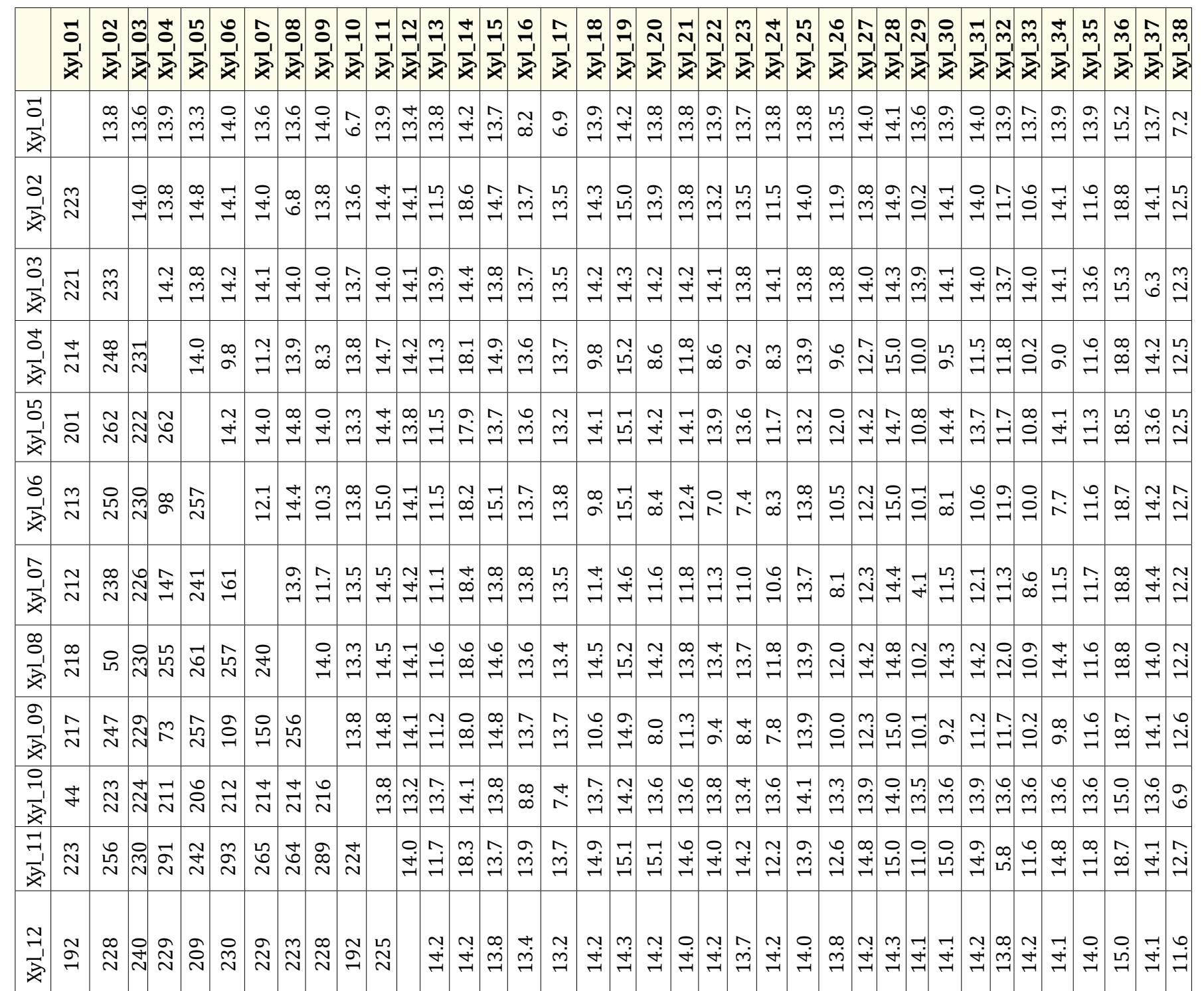

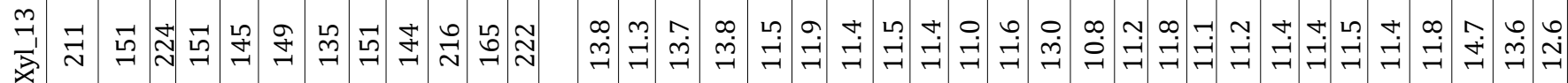

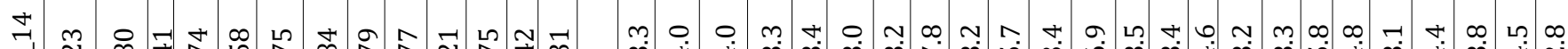
芳究

L

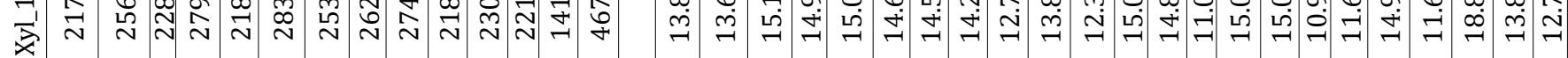

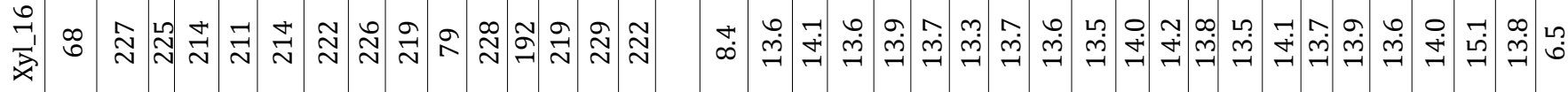




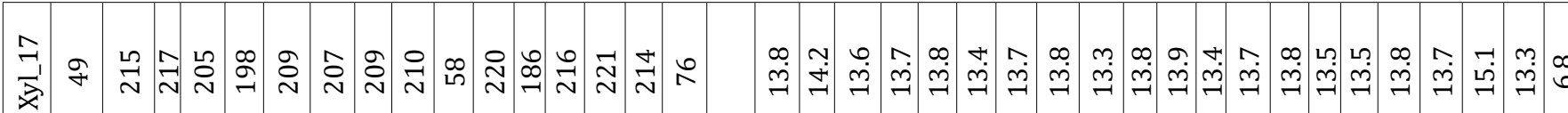

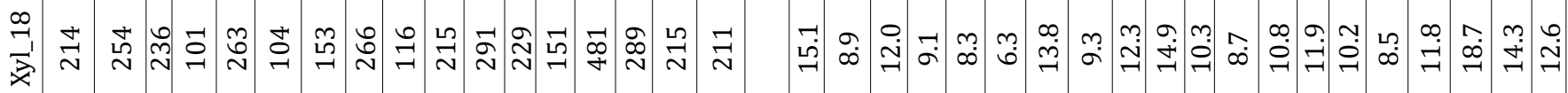

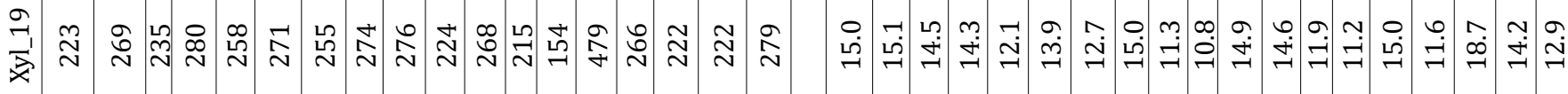

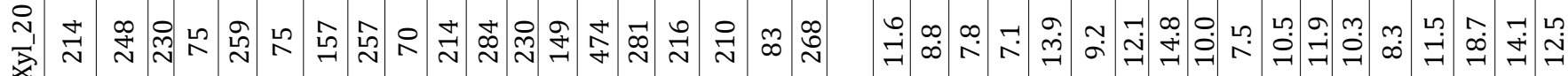

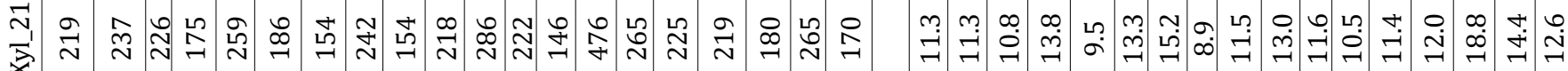

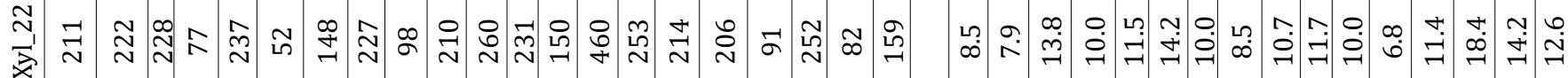

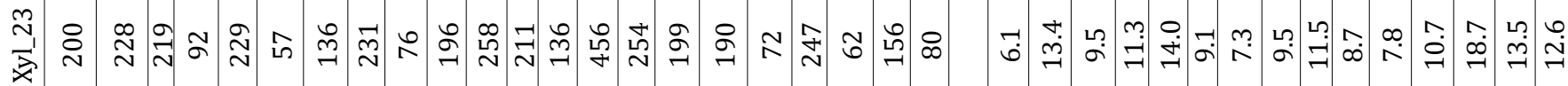

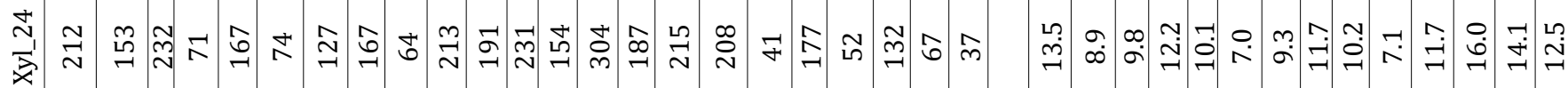

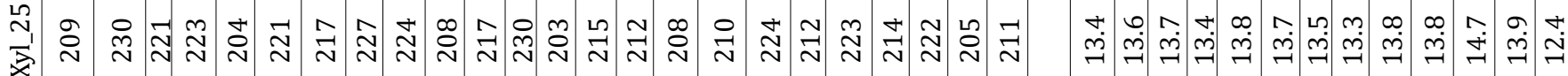

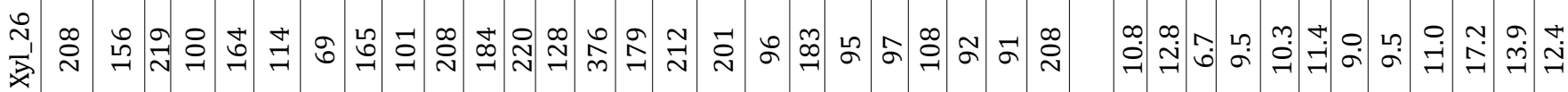

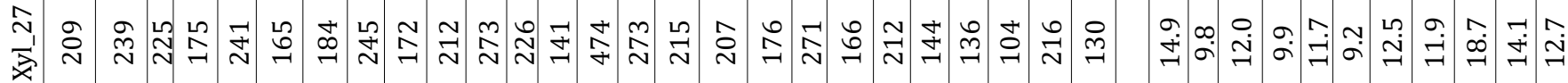

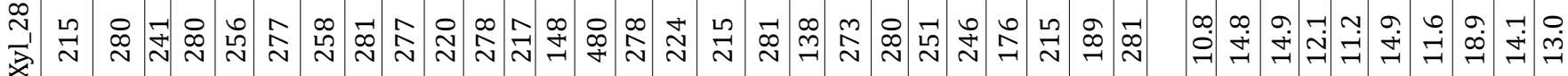

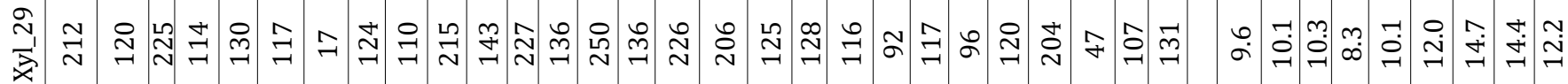

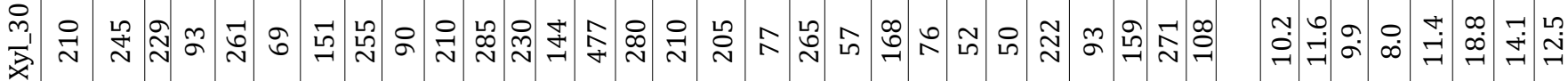




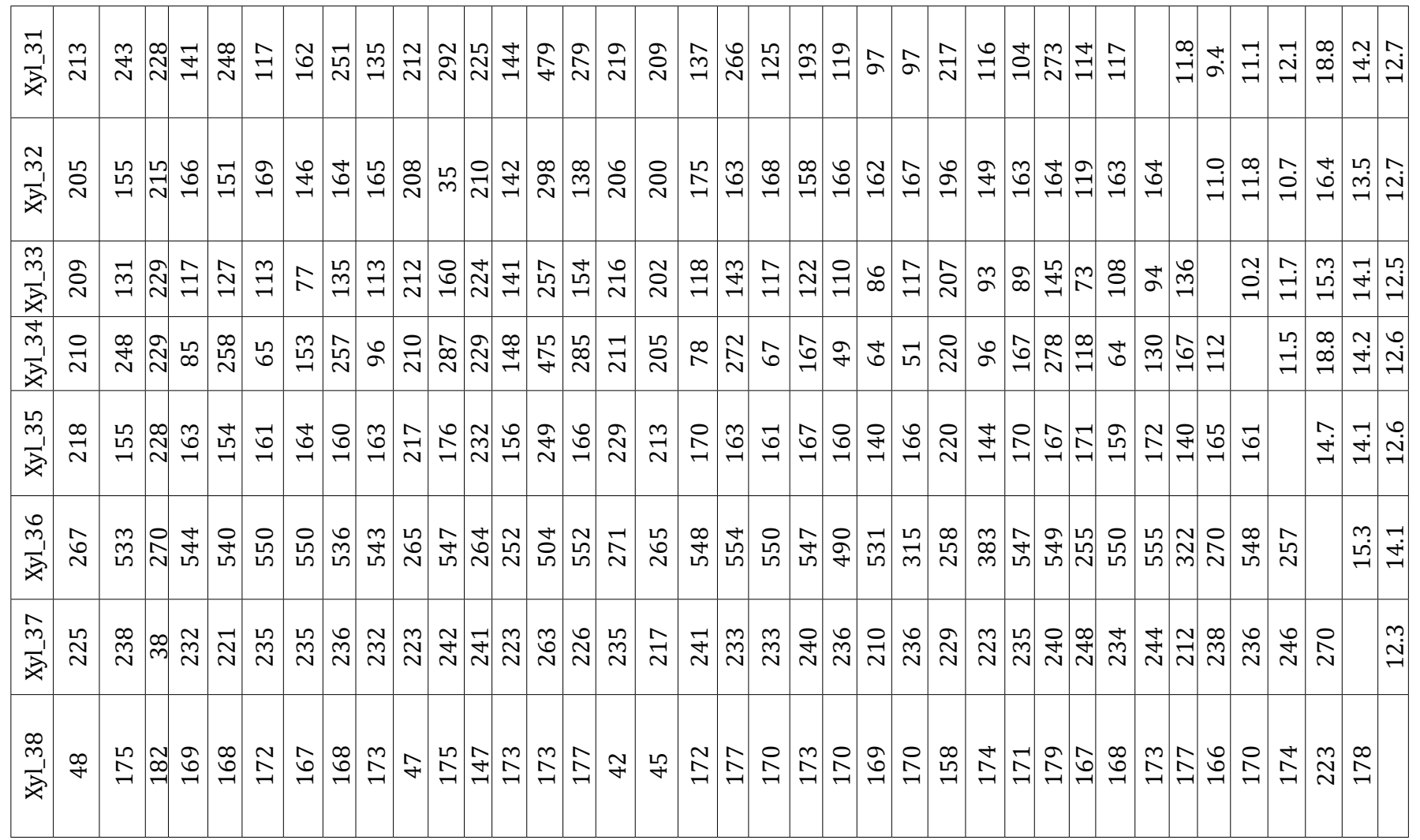

Table 3: Estimates of divergence between sequences, the number of amino acid differences per sequence from between sequences are shown. Standard error estimates are shown above the diagonal and all ambiguous positions were removed for each sequence pair (pairwise deletion).

\begin{tabular}{|l|c|c|}
\hline \multicolumn{1}{|c|}{ ID } & Identity & Acession \\
\hline Xyl_01 & $57,10 \%$ & AKY00358.1 \\
\hline Xyl_02 & $67,49 \%$ & AKY00359.1 \\
\hline Xyl_03 & $51,72 \%$ & ABZ41703.1 \\
\hline Xyl_04 & $66,33 \%$ & AKY00359.1 \\
\hline Xyl_05 & $64,42 \%$ & AKY00359.1 \\
\hline Xyl_06 & $66,24 \%$ & AKY00359.1 \\
\hline Xyl_07 & $68,79 \%$ & AKY00359.1 \\
\hline Xyl_08 & $67,10 \%$ & AKY00359.1 \\
\hline Xyl_09 & $66,33 \%$ & AKY00359.1 \\
\hline Xyl_10 & $54,91 \%$ & AKY00358.1 \\
\hline Xyl_11 & $64,46 \%$ & AKY00359.1 \\
\hline Xyl_12 & $49,43 \%$ & AKY00358.1 \\
\hline Xyl_13 & $57,01 \%$ & AKY00359.1 \\
\hline Xyl_14 & $42,53 \%$ & AER76185.1 \\
\hline Xyl_15 & $66,89 \%$ & AKY00359.1 \\
\hline Xyl_16 & $55,65 \%$ & AKY00374.1 \\
\hline Xyl_17 & $56,03 \%$ & AKY00358.1 \\
\hline Xyl_18 & $64,99 \%$ & AKY00390.1 \\
\hline Xyl_19 & $63,61 \%$ & AKY00359.1 \\
\hline Xyl_20 & $65,47 \%$ & AKY00359.1 \\
\hline
\end{tabular}

\begin{tabular}{|l|l|l|}
\hline Xyl_21 & $67,38 \%$ & AKY00359.1 \\
\hline Xyl_22 & $65,58 \%$ & AKY00359.1 \\
\hline Xyl_23 & $67,18 \%$ & AKY00359.1 \\
\hline Xyl_24 & $62,25 \%$ & AKY00359.1 \\
\hline Xyl_25 & $53,45 \%$ & ACP87342.1 \\
\hline Xyl_26 & $68,41 \%$ & AKY00390.1 \\
\hline Xyl_27 & $66,24 \%$ & AKY00359.1 \\
\hline Xyl_28 & $64,48 \%$ & AKY00359.1 \\
\hline Xyl_29 & $66,27 \%$ & AKY00359.1 \\
\hline Xyl_30 & $66,38 \%$ & AKY00359.1 \\
\hline Xyl_31 & $64,74 \%$ & AKY00359.1 \\
\hline Xyl_32 & $69,34 \%$ & AKY00390.1 \\
\hline Xyl_33 & $62,82 \%$ & AKY00359.1 \\
\hline Xyl_34 & $66,47 \%$ & AKY00359.1 \\
\hline Xyl_35 & $48,10 \%$ & AKY00359.1 \\
\hline Xyl_36 & $48,48 \%$ & AER76187.1 \\
\hline Xyl_37 & $59,87 \%$ & AKY00367.1 \\
\hline Xyl_38 & $57,70 \%$ & AKY00358.1 \\
\hline
\end{tabular}

Table 4: Percentage of identity between the 38 sequences identified in Metagenomic analysis and NCBI Patent database. 
proteins normally participate in specific processes and functions in biological systems [40], consequently making it difficult to obtain their soluble form.

In this sense, based on the set of in silico analyzes performed, 25 of the 38 sequences showed good characteristics for study, since the presence of the signal peptide is not an excluding criterion. Therefore, the choice for future in vitro analyzes was made based on the desirable characteristics present in the aforementioned sequences: absence of transmembrane helices, structural stability, thermal stability, identity with biotechnological application molecules, presence of domains of interest and difference in their amino acid sequences.

It is important to emphasize that one of the selected sequences has already presented promising laboratory results regarding its applicability and bifunctionality [41]. The other selected molecules will be investigated in future studies. Additionally, our work also contributes with a workflow that can be used to select enzymes of interest, starting from a database (Figure 1). It is important to note that this workflow can be used for other proteins and types of sequences once it is possible to analyze general characteristics of amino acid sequences.

\section{Conclusion}

The present work analyzed the diversity of sequences containing the GH10 domain present in the rumen of Nelore cattle. In addition to the identification of the 38 sequences, a workflow of in silico methodologies was suggested to select sequences that will guide future in vitro studies.

\section{Acknowledgements}

This work was supported by grants from grant 2018/12885-0, São Paulo Research Foundation (FAPESP), CNPq, and CAPES.

\section{Conflict of Interest}

The authors declare that there is not any conflict of interest within this paper.

\section{Bibliography}

1. Madhavan Aravind., et al. "Metagenome Analysis: A Powerful Tool for Enzyme Bioprospecting". Applied Biochemistry and Biotechnology 183.2 (2017): 636-651.

2. Handelsman Jo. "Metagenomics: Application of Genomics to Uncultured Microorganisms". Microbiology and Molecular Biology Reviews: MMBR 68.4 (2004): 669-685.
3. von Mering C., et al. "Quantitative Phylogenetic Assessment of Microbial Communities in Diverse Environments". Science (New York, N.Y.) 315.5815 (2007): 1126-1130.

4. Chistoserdova Ludmila. "Recent Progress and New Challenges in Metagenomics for Biotechnology". Biotechnology Letters 32.10 (2010): 1351-1359.

5. Sharma Hem Kanta., et al. "Biological Pretreatment of Lignocellulosic Biomass for Biofuels and Bioproducts: An Overview". Waste and Biomass Valorization 10.2 (2019): 235-251.

6. Lazuka Adèle., et al. "Anaerobic Lignocellulolytic Microbial Consortium Derived from Termite Gut: Enrichment, Lignocellulose Degradation and Community Dynamics". Biotechnology for Biofuels 11.1 (2018): 284.

7. Moraïs Sarah and Itzhak Mizrahi. "The Road Not Taken: The Rumen Microbiome, Functional Groups, and Community States". Trends in Microbiology 27.6 (2019): 538-549.

8. Deusch Simon., et al. "A Structural and Functional Elucidation of the Rumen Microbiome Influenced by Various Diets and Microenvironments". Frontiers in Microbiology 8 (2017).

9. Gruninger Robert J., et al. "Contributions of a Unique $\beta$-Clamp to Substrate Recognition Illuminates the Molecular Basis of Exolysis in Ferulic Acid Esterases". The Biochemical Journal 473.7 (2016): 839-849.

10. Ogunade Ibukun M., et al. "Effects of Live Yeast on Differential Genetic and Functional Attributes of Rumen Microbiota in Beef Cattle". Journal of Animal Science and Biotechnology 10.1 (2019): 68.

11. Collins T., et al. "Xylanases, Xylanase Families and Extremophilic Xylanases". Undefined (2005).

12. Gao Dahai., et al. "Hemicellulases and Auxiliary Enzymes for Improved Conversion of Lignocellulosic Biomass to Monosaccharides". Biotechnology for Biofuels 4 (2011): 5.

13. Beg Q K., et al. "Microbial Xylanases and Their Industrial Applications: A Review". Applied Microbiology and Biotechnology 56.3-4 (2001): 326-338.

14. de Freitas Caroline., et al. "Xylooligosaccharides Production Process from Lignocellulosic Biomass and Bioactive Effects". Bioactive Carbohydrates and Dietary Fibre 18 (2019): 100184. 
15. Venkateswar Rao Linga., et al. "Bioconversion of Lignocellulosic Biomass to Xylitol: An Overview”. Bioresource Technology 213 (2016): 299-310.

16. Jhamb Kamna and Debendra K Sahoo. "Production of Soluble Recombinant Proteins in Escherichia Coli: Effects of Process Conditions and Chaperone Co-Expression on Cell Growth and Production of Xylanase". Bioresource Technology 123 (2012): 135-143.

17. Hannig G and S C Makrides. "Strategies for Optimizing Heterologous Protein Expression in Escherichia Coli". Trends in Biotechnology 16.2 (1998): 54-60.

18. Peti Wolfgang and Rebecca Page. "Strategies to Maximize Heterologous Protein Expression in Escherichia Coli with Minimal Cost". Protein Expression and Purification 51.1 (2007): 1-10.

19. Leow Thean Chor., et al. "A Thermoalkaliphilic Lipase of Geobacillus Sp. T1". Extremophiles 11.3 (2007): 527-535.

20. Chang Catherine Ching Han., et al. "Bioinformatics Approaches for Improved Recombinant Protein Production in Escherichia Coli: Protein Solubility Prediction". Briefings in Bioinformatics 15.6 (2014): 953-962.

21. Gaspar Paulo., et al. "EuGene: Maximizing Synthetic Gene Design for Heterologous Expression". Bioinformatics 28.20 (2012): 2683-2684.

22. Powell Sean., et al. "EggNOG v4.0: Nested Orthology Inference across 3686 Organisms". Nucleic Acids Research 42 (2014): D231-239.

23. Thompson J D., et al. "CLUSTAL W: Improving the Sensitivity of Progressive Multiple Sequence Alignment through Sequence Weighting, Position-Specific Gap Penalties and Weight Matrix Choice". Nucleic Acids Research 22.22 (1994): 4673-4680.

24. Finn Robert D., et al. "Pfam: The Protein Families Database". Nucleic Acids Research 42 (2014): D222-230.

25. Gasteiger Elisabeth., et al. "Protein Identification and Analysis Tools on the ExPASy Server". The Proteomics Protocols Handbook, edited by John M. Walker, Humana Press (2005): 571607.

26. Almagro Armenteros José Juan., et al. "SignalP 5.0 Improves Signal Peptide Predictions Using Deep Neural Networks". Nature Biotechnology 37.4 (2019): 420-423.
27. Krogh A., et al. "Predicting Transmembrane Protein Topology with a Hidden Markov Model: Application to Complete Genomes". Journal of Molecular Biology 305.3 (2001): 567-580.

28. Huang Le., et al. "DbCAN-Seq: A Database of CarbohydrateActive Enzyme (CAZyme) Sequence and Annotation". Nucleic Acids Research 46.D1 (2018): D516-521.

29. Altschul S F., et al. "Basic Local Alignment Search Tool”. Journal of Molecular Biology 215.3 (1990): 403-410.

30. Larkin M A., et al. "Clustal W and Clustal X Version 2.0". Bioinformatics (Oxford, England) 23.21 (2007): 2947-2948.

31. Kumar Sudhir., et al. "MEGA X: Molecular Evolutionary Genetics Analysis across Computing Platforms". Molecular Biology and Evolution 35.6 (2018): 1547-1549.

32. Saitou N and M Nei. "The Neighbor-Joining Method: A New Method for Reconstructing Phylogenetic Trees". Molecular Biology and Evolution 4.4 (1987): 406-425.

33. Felsenstein Joseph. "CONFIDENCE LIMITS ON PHYLOGENIES: AN APPROACH USING THE BOOTSTRAP”. International Journal of Organic Evolution 39.4 (1985): 783-791.

34. Masatoshi Nei and Sudhir Kumar. Molecular Evolution and Phylogenetics. Oxford University Press (2000).

35. Letunic Ivica and Peer Bork. "Interactive Tree Of Life (ITOL) v5: An Online Tool for Phylogenetic Tree Display and Annotation". Nucleic Acids Research (2021).

36. Kmezik Cathleen., et al. "A Polysaccharide Utilization Locus from the Gut Bacterium Dysgonomonas Mossii Encodes Functionally Distinct Carbohydrate Esterases". Journal of Biological Chemistry 296 (2021): 100500.

37. Qiao Weibo., et al. "Biochemical Characterization of a Novel Thermostable GH11 Xylanase with CBM6 Domain from Caldicellulosiruptor Kronotskyensis". Journal of Molecular Catalysis B: Enzymatic 107 (2014): 8-16.

38. Mewis Keith., et al. "Dividing the Large Glycoside Hydrolase Family 43 into Subfamilies: A Motivation for Detailed Enzyme Characterization". Applied and Environmental Microbiology 82.6 (2016): 1686-1692.

39. Glasgow Evan., et al. "Multifunctional Cellulases Are Potent, Versatile Tools for a Renewable Bioeconomy". Current Opinion in Biotechnology 67 (2021): 141-148. 
40. Möller Steffen., et al. "Evaluation of Methods for the Prediction of Membrane Spanning Regions". Bioinformatics 17.7 (2001): 646-653.

41. Pavarina Gabriella Cavazzini., et al. "Characterization of a New Bifunctional Endo-1,4- $\beta$-Xylanase/Esterase Found in the Rumen Metagenome". Scientific Reports 11.1 (2021): 10440.

\section{Volume 4 Issue 7 July 2021}

(C) All rights are reserved by Adriano Marques

Gonçalves., et al. 